\title{
CARACTERÍSTICAS DE ACIDENTES POR TRANSPORTE TERRESTRE ATENDIDOS EM HOSPITAIS PÚBLICOS
}

\author{
Jéssica Rodolfo Campos ${ }^{1}$, Eliane Regina Pereira do Nascimento², Patrícia Madalena Vieira \\ Hermida $^{3}$, Sabrina Guterres da Silva Galetto ${ }^{4}$, Maíra Antonello Rasia ${ }^{5}$, NatyeleRippel Silveira ${ }^{6}$
}

\begin{abstract}
RESUMO: Objetivo: caracterizar os acidentes por transporte terrestre atendidos em hospitais públicos de referência no Estado de Santa Catarina. Método: pesquisa descritiva e quantitativa. Os dadosdos acidentes foram coletados, de julho a setembro de 2015, mediante formulário estruturado. Constituíram a amostra 139 prontuários de duas emergências. Na análise,aplicou-se a estatística descritiva. Resultados: os acidentes envolveram principalmente homens ( $\mathrm{n}=112 ; 80,6 \%)$, de 20 a 29 anos $(n=53 ; 38,2 \%)$. Destacaram-se os acidentes de colisão carro/moto ( $n=44 ; 31,7 \%)$ e ocorridos nas sextas-feiras $(n=27 ; 19,4 \%)$ à noite $(n=11 ; 7,9 \%)$. O segmento corpóreo mais atingidoisoladamente foram os membros inferiores ( $n=49 ; 35,2 \%)$. Na maioria dos acidentes ( $n=95 ; 68,3 \%)$,o paciente ficou hospitalizadopor, no mínimo,24 horas. Conclusão: as medidas preventivas dos acidentes por transporte terrestre deverão se voltar, sobretudo, aos homens, jovens, que utilizam automóvel e motocicleta, destacando-se as ações de fiscalização do trânsito próximas ao final de semana e de estímulo ao uso de equipamentos de segurança.
\end{abstract}

DESCRITORES: Acidentes de trânsito; Ferimentos e lesões; Serviços médicos de emergência; Hospitais públicos.

\section{CHARACTERISTICS OF ROAD TRAFFIC ACCIDENTS BASED ON PUBLIC HOSPITAL SERVICES}

\begin{abstract}
Objective: to characterize road traffic accidents based on the services provided in public reference hospitals of the state of Santa Catarina. Method: descriptive, quantitative research. Data of the accidents were collected from July to September 2015 through the use of a structured form. The sample consisted of 139 medical records of two emergency units. Descriptive statistics was applied in the analysis. Results: the accidents mainly involved men $(n=112 ; 80.6 \%)$, aged from 20 to 29 years ( $n=53 ; 38.2 \%$ ). There was a predominance of car/ motorcycle accidents $(n=44 ; 31.7 \%)$ on Fridays $(n=27 ; 19.4 \%)$ in the evening $(n=11 ; 7.9 \%)$. Lower limbs represented the most commonly body segment individually affected $(n=49 ; 35.2 \%)$. In most accidents $(n=95 ; 68.3 \%)$ the patient remained hospitalized for at least 24 hours. Conclusion: preventive measures for road traffic accidents should be especially focused on young men that use cars and motorcycles, with an emphasis on traffic control actions on the eve of weekends and actions promoting the use of safety equipment.

DESCRIPTORS: Accidents, traffic; Wounds and injuries; Emergency medical services; Public hospitals.
\end{abstract}

\section{CARACTERÍSTICAS DE ACCIDENTES DE TRANSPORTE TERRESTRE ATENDIDOS EN HOSPITALES PÚBLICOS}

RESUMEN: Objetivo: Caracterizar los accidentes de transporte terrestre atendidos en hospitales públicos de referencia del Estado de Santa Catarina. Método: Investigación descriptiva, cuantitativa. Datos de accidentes recolectados de julio a setiembre de 2015, mediante formulario estructurado. Muestra constituida por 139 historias clínicas de dos servicios de urgencias. Los datos recolectados fueron analizados por estadística descriptiva. Resultados: Los accidentes involucraron especialmente a hombres ( $n=112 ; 80,6 \%)$, de 20 a 29 años ( $n=53 ; 38,2 \%)$. Prevalecieron accidentes de colisión automóvil/motocicleta $(n=44 ; 31,7 \%)$ sucedidos en viernes $(n=27 ; 19,4 \%)$, de noche $(n=11 ; 7,9 \%)$. La zona corporal más afectada individualmente fueron los miembros inferiores $(n=49 ; 35,2 \%)$. En la mayoría de los accidentes ( $n=95$; $68,3 \%)$, el paciente quedó internado al menos por 24 horas. Conclusión: Las medidas preventivas para accidentes de transporte terrestre deberán orientarse particularmente a hombres jóvenes, usuarios de automóviles y motocicletas, destacándose acciones de control de tránsito cercanas al fin de semana, y de promoción del uso de equipos de seguridad.

DESCRIPTORES: Accidentes de tránsito; Heridas y lesiones; Servicios médicos de urgencia; Hospitales públicos.

${ }^{1}$ Enfermeira. Graduada em Enfermagem na Universidade Federal de Santa Catarina. Florianópolis, SC, Brasil.

${ }^{2}$ Enfermeira. Doutora em Enfermagem. Docente do Departamento de Enfermagem e do Programa de Pós-Graduação em Enfermagem da Universidade Federal de Santa Catarina. Florianópolis, SC, Brasil.

${ }^{3}$ Enfermeira. Doutora em Enfermagem. Pós-doutoranda em Enfermagem do Programa de Pós-Graduação em Enfermagem da Universidade Federal de Santa Catarina. Florianópolis, SC, Brasil.

${ }^{4}$ Enfermeira. Doutoranda em Enfermagem do Programa de Pós-Graduação em Enfermagem da Universidade Federal de Santa Catarina. Florianópolis, SC, Brasil.

${ }^{5}$ Enfermeira. Mestre Multidisciplinar em Saúde. Responsável Técnica de Enfermagem da Associação de Pais e Amigos dos Excepcionais de Florianópolis. Florianópolis, SC, Brasil.

${ }^{6}$ Enfermeira. Mestre em Enfermagem pela Universidade Federal de Santa Catarina. Florianópolis, SC, Brasil. 


\section{INTRODUÇÃO}

Muitas pessoas que sobrevivem aos Acidentes por Transporte Terrestre (ATT) adquirem incapacidades temporárias ou permanentes ${ }^{(1)}$. A Organização Mundial da Saúde estima que, até 2030, os ATT sejam a terceira dentre todas as causas de incapacidades na população mundial ${ }^{(2)}$. Metade de todas as mortes decorrentes de lesões por ATT é compostapor pedestres, ciclistas ou motociclistas. Os jovens de 15 a 29 anos são os mais acometidos e 90\% das mortes ocorrem em países de baixa e média renda. Os acidentes, além de provocar mortes e incapacidades, geram impacto substancial às economias nacionais e às famílias das pessoas acometidas ${ }^{(3)}$.

Nesse sentido, os ATT se mostram como um importante problema de saúde pública ${ }^{(1)}$. Em se tratando da realidade brasileira, a taxa de mortalidade por ATT, em 2013, foi de 21 óbitos por 100 mil habitantes, o que resultou, em todo o país, em mais de um milhão de anos potenciais de vida perdidos ${ }^{(4)}$. Quanto à taxa de internação pela mesma causa, correspondeu a 85,0 por 100 mil habitantes, alcançando um gasto total que ultrapassou os $\mathrm{R} \$ 230$ milhões financiados pelo Sistema Único de Saúde ${ }^{(5)}$. Dentre os Estados brasileiros, Santa Catarina é o segundo em número de mortes por ATT. Em 2012,somente nas rodovias estaduais, foram 355 óbitos no momento do acidente ${ }^{(6)}$.

Ao considerar que o setor de emergência hospitalar é a porta de entrada de pessoas com situações agudas de saúde, dentre elas, aquelas acometidas pelos ATT, questiona-se: Quais as características dos acidentes por transporte terrestre nos atendimentos realizados em hospitais públicos de referência do Estado de Santa Catarina? Para responder a esse questionamento, traçou-se como objetivo: caracterizar os acidentes por transporte terrestre atendidos em hospitais públicos de referência no Estado de Santa Catarina.

\section{- MÉTODO}

Trata-se de uma pesquisa descritiva, com abordagem quantitativa, desenvolvida em unidades de emergência de dois hospitais públicos de referência em trauma localizados na região da Grande Florianópolis, Santa Catarina. Essa região engloba 22 municípios, dentre eles, São José e Florianópolis, onde o estudo foi realizado.

Estabeleceram-se como critérios de inclusão para a realização da pesquisa: prontuários de pacientesenvolvidos em ATT ocorridos no período de 02 de julho a 18 de setembro de 2015, com idade mínima de 15 anos, atendidos na emergência dos hospitais em questão e que permaneceram ou não hospitalizados. Excluíram-se os prontuários dos pacientes que não estavam no hospital para autorizar a consulta ao prontuário. Optou-se pela amostragem não probabilística, por conveniência, o que envolveu o recrutamento, nos dois hospitais, do prontuário dos pacientes de ATT que atenderam aos critérios de elegibilidade ao longo do período de coleta de dados. A amostra foi constituída por 139 prontuários eletrônicos.

A coleta dos dados foi realizada por uma mesma pesquisadora em ambos os hospitais, de segunda a sexta-feira, no período da manhã ou tarde. Para identificar os pacientes de ATT, consultou-seo enfermeiro do plantão no setor de Emergência e/ou o sistema de registro. A seguir, o paciente, ao ser localizado e orientado sobre o estudo, autorizavaa consulta ao prontuário, que foi acessado para o registro dos dadosem um formulário eletrônico previamente elaborado para esta investigação.

As variáveis contempladas no formulário foram: idade; sexo; tipo de acidente; dia da semana e turno do dia em que ocorreu o acidente; segmentos corpóreos afetados isoladamente; número de segmentos afetados concomitantemente e tempo de permanência hospitalar.

Os dados foram armazenados, organizados e analisados utilizando-se o SoftwareEpi Info, versão 3.5.2., sendo aplicada a estatística descritiva simples (frequências absoluta e relativa). Para a organização e a análise dos dados referentes ao tempo de permanência no hospital, considerou-se, como internação, o período mínimo de 24 horas. 
A pesquisa foi aprovada pelo Comitê de Ética em Pesquisa da Universidade Federal de Santa Catarina sob o Parecer n.o 1.137.760. Todos os participantes do estudo assinaram o Termo de Consentimento Livre e Esclarecido. No caso de pacientes com idade inferior a 18 anos, o assentimento foi do adulto responsável legal.

\section{RESULTADOS}

Os atendimentos por ATT nos hospitais evidenciaram que os pacientesforam, em sua maioria, do sexo masculino $(n=112 ; 80,6 \%)$, sendo as faixas etárias mais acometidas de 20 a 29 anos $(n=53 ; 38,2 \%)$ e de 30 a $39(n=42 ; 30,2 \%)$ que, juntas, totalizam $68,4 \%$ dos atendimentos.

Em relação aos segmentos corpóreos atingidos isoladamente em cada um dos pacientes destacaramse os membros inferiores $(n=43 ; 30,9 \%)$. Contudo, houve pacientes que apresentaram lesões em, pelo menos, dois segmentos corporais $(n=53 ; 38,1 \%)$, predominando aqueles com dois segmentos afetados $(\mathrm{n}=37 ; 26,6 \%)$.Quanto ao tempo de permanênciado paciente no hospital, a maioria permaneceu internada por, no mínimo,24 horas(n=95; 68,3\%), conforme a tabela 1.

Tabela 1 - Características dos atendimentos porAcidentes porTransporte Terrestre $(n=139)$ em hospitais de referência. Florianópolis, SC, Brasil, 2015. (continua)

\begin{tabular}{|c|c|c|}
\hline Variável & $\mathbf{n}$ & $\%$ \\
\hline \multicolumn{3}{|l|}{ Sexo } \\
\hline Masculino & 112 & 80,6 \\
\hline Feminino & 27 & 19,4 \\
\hline \multicolumn{3}{|l|}{ Faixa etária (em anos) } \\
\hline$<20$ & 08 & 5,8 \\
\hline 20 a 29 & 53 & 38,2 \\
\hline 30 a 39 & 42 & 30,2 \\
\hline 40 a 49 & 22 & 15,8 \\
\hline 50 a 59 & 12 & 8,6 \\
\hline$\geq 60$ & 02 & 1,4 \\
\hline \multicolumn{3}{|c|}{ Segmento corpóreo atingido isoladamente } \\
\hline Membros inferiores & 49 & 35,2 \\
\hline Membros superiores & 13 & 9,4 \\
\hline Tórax & 12 & 8,6 \\
\hline Abdome & 05 & 3,6 \\
\hline Cabeça & 04 & 2,9 \\
\hline Pescoço & 03 & 2,2 \\
\hline \multicolumn{3}{|c|}{ Número de segmentos corpóreos atingidos concomitantemente } \\
\hline Dois segmentos & 37 & 26,6 \\
\hline Três segmentos & 13 & 9,4 \\
\hline Quatro segmentos & 02 & 1,4 \\
\hline Cinco ou mais segmentos & 01 & 0,7 \\
\hline \multicolumn{3}{|c|}{ Tempo de permanência no hospital (em dias) } \\
\hline$<1^{*}$ & 40 & 28,8 \\
\hline 1 a 7 & 37 & 26,6 \\
\hline
\end{tabular}




\begin{tabular}{llc}
\hline 8 a 14 & 31 & 22,3 \\
\hline 15 a 21 & 10 & 7,2 \\
\hline 22 a 28 & 08 & 5,8 \\
\hline 29 a 35 & 02 & 1,4 \\
\hline$>35$ & 07 & 5 \\
\hline Não identificado & 04 & 2,9 \\
\hline
\end{tabular}

Legenda: ${ }^{*}<1=$ menos que 24 horas.

Constatou-se que os principais tipos de ATT foram: colisão carro/moto ( $n=44 ; 31,7 \%)$; queda de motocicleta $(n=24 ; 17,3 \%)$; acidente de motocicleta não especificado $(n=13 ; 9,4 \%)$; atropelamento $(\mathrm{n}=10 ; 7,2 \%)$ e colisão moto/outro veículo (caminhão, van escolar, trator ou ambulância), $n=9$; $6,5 \%$, conforme a figura 1 .

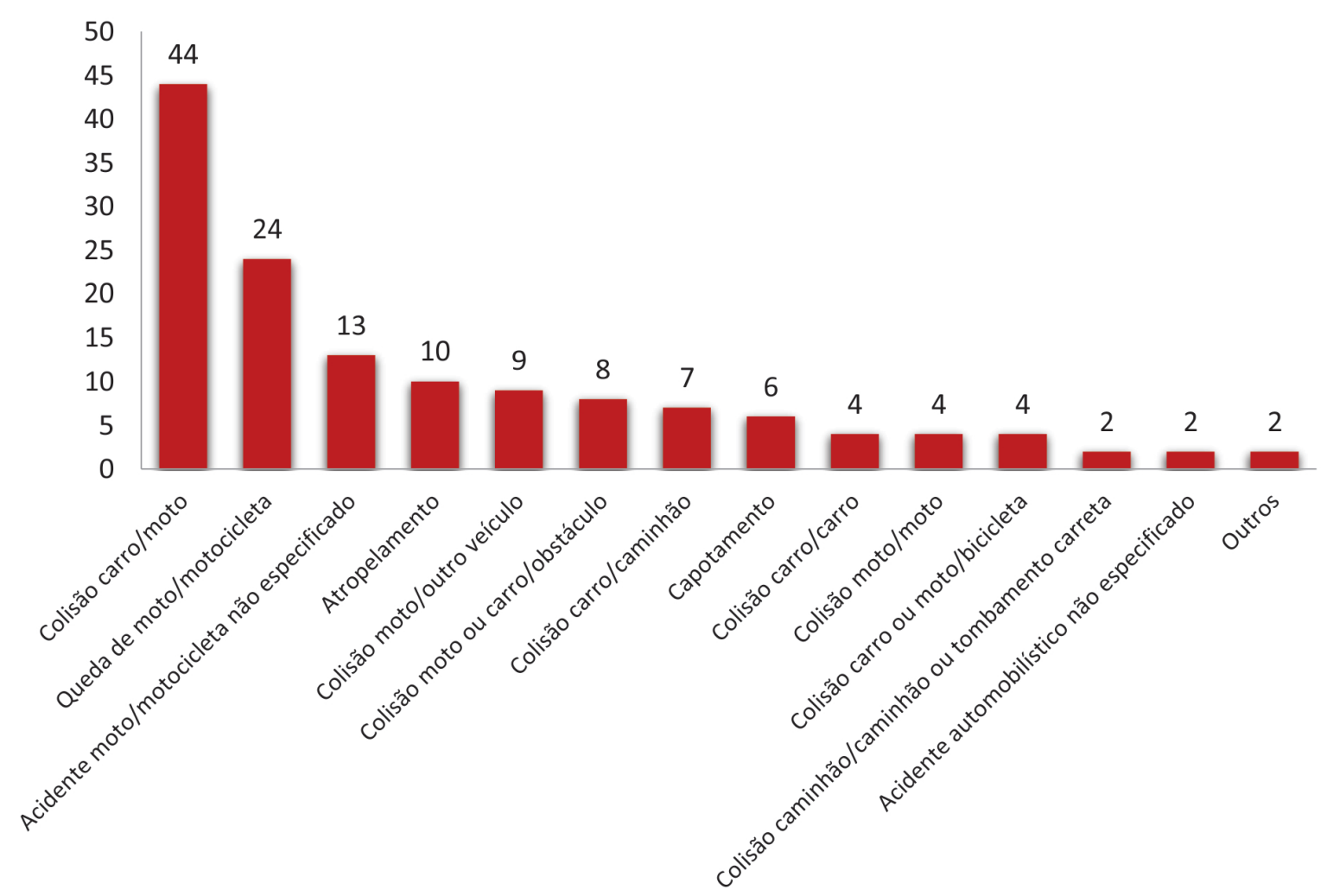

Figura 1 - Frequência absoluta $(n=139)$ dos tipos de Acidente por Transporte Terrestre atendidos em hospitaisde referência. Florianópolis, SC, Brasil, 2015

Quanto ao turno de ocorrência dos ATT, houve mais acidentes no período da tarde $(n=50 ; 36 \%)$ e da noite $(n=36 ; 25,9 \%)$ totalizando $61,9 \%$ dos acidentes. O dia da semana com maior número de acidentes foi sexta-feira $(n=27 ; 19,4 \%)$, seguido do sábado $(n=24 ; 17,3 \%)$ e da segunda-feira $(n=24$; $17,3 \%)$. Chama a atenção que a frequência de acidentes na madrugada igualou-se de terça a sexta-feira $(n=1 ; 0,7 \%)$. Considerando-se o dia da semana e o turno do dia, concomitantemente, o maior número de acidentes ocorreu na sexta-feira à noite $(n=11 ; 7,9 \%)$, conforme a figura 2 . 


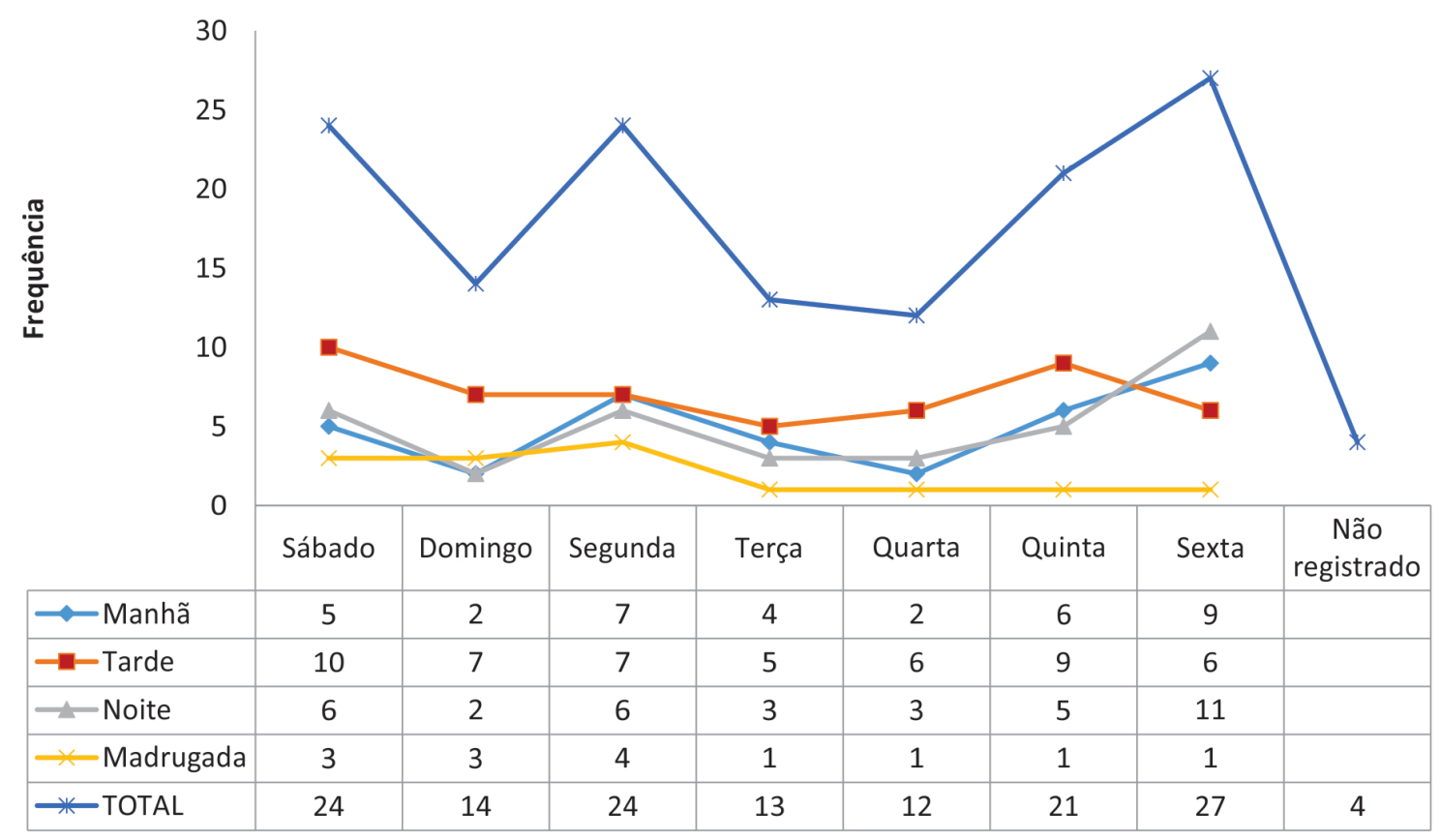

Figura 2 - Dia da semana de ocorrência do acidente ( $n=139)$ segundo o período do dia. Florianópolis, SC, Brasil, 2015

\section{DISCUSSÃO}

Os resultados evidenciam que os pacientes envolvidos nos ATTse assemelhamàqueles identificados em outros estudos, sendo os mais acometidos pelos acidenteshomens jovens ${ }^{(7-11)}$. No Irã e Sri Lanka, por exemplo, a maioria dos envolvidos era do sexo masculino, sendo que 32,5\% e $26 \%$ tinham entre 20 e 29 anos, respectivamente ${ }^{(11-12)}$, faixa etária que, neste estudo,correspondeu a 38,2\% dos pacientes.

Estegrupo populacional está mais propenso aos ATT devido à maior exposição às situações de risco como inexperiência ao volante, condução além dos limites de velocidade permitidos, seguida da exposição mais arriscada na direção de veículos e consumo, por vezes, abusivo de bebidas alcoólicas e direção após este consumo, dentre outros ${ }^{(10)}$. Desse modo, reduzir os ATT entre os jovens requer o uso de estratégias peculiares como medidas de educação para a mudança de hábitos e comportamentos e intensificação nas fiscalizações com ações articuladas entre diversos setores ${ }^{(13)}$.

Nos pacientes que tiveram um único segmento corpóreo afetado, predominaramas lesões de membros inferiores, achado também identificado empesquisasimilarrealizada no Serviço de Atendimento Móvel de Urgência ${ }^{(9)}$. Diferentemente, no Irã, foram os membros superiores os mais atingidos entre os pacientes, seguidos da cabeça e pescoço(11), enquanto, na Nigéria, a cabeça e pescoço ocuparam a primeira posição entre os segmentos corpóreos atingidos ${ }^{(14)}$, o que pode estar associado ao não uso do cinto de segurança e capacete, equipamentos de utilização obrigatória no Brasil. No Quênia, por exemplo,o uso de capacete entre motociclistas se mostrou associado a uma redução significativa de lesões na cabeça ${ }^{(15)}$.

Os pacientes que apresentaram lesões em mais de um segmento corporalrepresentaram menos da metade da amostra, aspecto que difere de outros estudos. No âmbito nacional e internacional, foi revelado que a maioria dos pacientes de ATT teve múltiplos segmentos atingidos ${ }^{(9,11)}$ e que a presença de várias lesões é preditora de mortalidade hospitalar ${ }^{(11)}$.Pode-se inferir que, nos ATT, os segmentos afetados e a quantidade destes têm relação com o tipo de acidente.

Nesse sentido, pesquisas que envolveram somente acidentes motociclísticos mostraram predomínio de lesões em membros inferiores ${ }^{(8,16)}$. Estudo que incluiu somente pacientes com traumatismo cranioencefálico, decorrentes de acidentes de motocicleta, identificou que a maioria teve lesão,também,em outros segmentos corporais ${ }^{(17)}$.Isso sinaliza que a exposição das pessoas, própria dos acidentes motociclísticos, pode causar lesões em múltiplos segmentos. 
Sobre as internações por acidentes de transporte, observa-se o aumento nas últimas décadas, principalmente envolvendomotociclistas ${ }^{(18)}$.A frequência das internações por ATT nas instituições investigadas (a maioria permaneceu internada por, no mínimo, 24 horas: n=95; 68,3\%) divergede um estudo também realizado em Santa Catarina cujopercentual de internação foi de $33 \%{ }^{(7)}$.

Os achados podemindicar a relação com o tipo de acidente e agravidadeda lesão causadaconsiderandose quea maioria dos pacientes permaneceu no hospital por, no mínimo,24 horas. Acondição em que a pessoa se encontra no trânsito (ciclista, pedestre, etc.) determina a gravidade das lesões ${ }^{(19)}$.

Na cidade de São Paulo, a frequência das internações com duração de zero a sete dias, ocorridas por acidentes de motocicleta, representou $76,9 \%$ dos $\operatorname{casos}^{(20)}$, percentual maior que o identificado neste estudo, quefoi de 55,4\%, considerando os pacientes que receberam alta em menos de 24 horas e os que permaneceram no hospital de um até sete dias,em decorrência de todos os tipos de ATT. Em Minas Gerais, os ATT registraram, em média, 6,1 dias de internação por paciente. Os pacientes com maior período de hospitalização (permanência média de 6,4 dias) foram os pedestres, devido a atropelamento, e os ocupantes de veículos, apresentando os maiores coeficientes de letalidade $(4,6$ e $5,1 \%$, respectivamente $)^{(18)}$.

Média de internação semelhante (6,3 dias) foi evidenciada, em todo território nacional, quando foram registradas, em 2013, 170.805 hospitalizações decorrentes de ATT, representando um problema de elevada magnitude e de gastos expressivos para o setor desaúde ${ }^{(5)}$. No mesmo ano, resultado menos favorável em relação ao período de hospitalização foi revelado na Espanha, cuja média de internação decorrente de ATT foi de 8,65 dias ${ }^{(21)}$.

Constatou-se, nos 139 prontuários pesquisados, que os principais tipos de ATT envolveram motocicleta, os quais foram, na sequência: colisão carro/moto; queda de motocicleta e acidente de motocicleta não especificado. Em consonância com estes achados, estudorevelou a colisão entre veículos como o tipo de acidente predominante, sendo a motocicleta o principal veículo envolvido nos acidentes com vítimas ${ }^{(22)}$.Muito embora a grande maioria dos usuários de motocicleta considere que está em alto risco para se envolver em acidentes, utiliza este veículo, principalmente, para o lazer e o deslocamento ao trabalho ou escola ${ }^{(23)}$.

As colisões entre carros e motocicletastambém são identificadas como predominantes nolrã ${ }^{(11)}$, com estatística semelhanteà desta pesquisa. No Brasil, outro estudo revelou esse tipo de acidente como o principal, porém, com quantitativo mais elevado, evidenciando que os dados encontrados são condizentes com as distintas realidades rodoviárias do país ${ }^{(24)}$.

A queda de moto, como neste estudo, ocupou a segunda posição no número de ATT ocorridos no Piauí ${ }^{(24)}$. Dentre os motivos que levam a esse tipo de acidente estão os conflitos ocorridos no trânsito com veículos mais suscetíveis ${ }^{(19)}$.

Quanto ao turno de ocorrência dos ATT, destacaram-se os acidentes ocorridos no período da tarde,com maior frequência, seguidos dosà noite.Resultado inverso (noite e tarde) foi identificado no país em investigações similares que envolveram somente acidentes motociclísticos ${ }^{(17,25)}$, assim como no âmbito internacional, cuja pesquisa englobou diferentes tipos de $\mathrm{ATT}^{(11)}$. O cansaço dos condutores, gerado ao longo do dia, o fluxo de veículos após expedientes de trabalho, a visibilidade prejudicada pela iluminação diminuída com o anoitecer, a redução da fiscalização por parte da polícia, além do abuso de álcool e drogas ilícitas são causas do maior número de acidentes nesses períodos do dia ${ }^{(25)}$.

Dado peculiar relativo aos ATT se refere às ocorrências que aconteceramna madrugada. O menor quantitativo de acidentes nesse período pode estar relacionado especialmente ao menor tráfego de veículos e consequentes colisões entre os mesmos.

No tocante ao dia da semana das ocorrências, maior frequência foi revelada na sexta-feira, seguida do sábado e segunda-feira. Cabe registrar que Florianópolis é uma cidade turística com atrativos naturais e, também, boêmios, com uma grande variedade de bares e casas noturnas que estimulam a vida noturna, principalmente na sexta-feira e sábado, o que contribuiria com o quantitativo mais elevado de acidentes nesses dias.

Comparativamente, outras pesquisas desenvolvidas no Brasil apontaram o final de semana (sábado e domingo) como os dias de maior ocorrência de $\mathrm{ATT}^{(8-9)}$, o que remete ao trânsito livre, ausência de 
congestionamentos e ingestão de bebidas alcoólicas ${ }^{(19)}$.Estudo realizado em Nepal evidenciou que os acidentes acontecem principalmente no sábado, o que também sugere ligação com o fato de as pessoas deixarem suas casas nesse dia para diversos fins, uma vez que ele representa a única folga da semana no país ${ }^{(26)}$.

Sobre as limitações desta investigação, os autores reconhecem que o tipo de amostragem adotada e a definição de um período para a coleta dos dados podem limitar a generalização dos resultados, o que exigiu cautela dos pesquisadores na interpretação dos achados e na elaboração das conclusões. Contudo, o fato dos hospitais participantes da pesquisa atuarem como referência no Estado, no atendimento às pessoas acometidas de trauma por ATT, indicaque a amostra empregada caracteriza esse tipo de acidente, especialmente em relação àqueles ocorridos na região da Grande Florianópolis.

\section{CONCLUSÃO}

Os ATT nos atendimentos realizados em hospitais de referência de Santa Catarina englobaram principalmente homens, jovens, envolvidos em acidentes motociclísticoscom quedaou colisão moto/ carro, sendo esse último o tipo predominante. Os membros inferiores foram os segmentos corpóreos mais atingidosisoladamente e destacaram-se os pacientes que permaneceram internados no hospital por, pelo menos,24 horas.Quanto ao turno e dia das ocorrências, identificou-se maior quantitativo de acidentes na sexta-feira à noite.

A caracterização dos acidentes por transporte terrestre no cenário hospitalar pode auxiliar no planejamento da assistência de Enfermagem e da saúde de uma maneira em geral. No campo das políticas públicas, é fundamental para subsidiar o planejamentodestas, a fim de se tornarem mais efetivas, com consequente redução da morbimortalidade poresse tipo de acidente.

Nesse sentido, julga-se que as medidas preventivas dos acidentes devemse voltar, sobretudo, aos homens, jovens, que utilizam automóvel e motocicleta, destacando-se as ações de fiscalização do trânsito próximas ao final de semana e de estímulo ao uso de equipamentos de segurança para a proteção da integridade corporal.

Sugerem-se, para novas investigações, estudos longitudinais e correlacionais sobre a temática a fim de avaliar a tendência dos ATT em Santa Catarina e os fatores associados, respectivamente.

\section{REFERÊNCIAS}

1. World Health Organization (WHO). Injuries and violence: the facts 2014. [Internet] Geneva: WHO; 2014 [acesso em 10 dez 2017]. Disponível: http://apps.who.int/iris/bitstream/10665/149798/1/9789241508018_eng.pdf?ua=1 \&ua=1.

2. Schemitsch EH, Bhandari M. Editorial note: a decade of collaboration and action. J.Orthop. Trauma. [Internet] 2014;28(6) [acesso em 5 jun 2017]. Disponível: http://dx.doi.org/10.1097/BOT.0000000000000102.

3. Organização Mundial da Saúde (OMS). Gestão das Doenças Transmissíveis, Incapacitantes, Violência e Prevenção de Traumatismos (NVI). Relatório global sobre o estado da segurança viária 2015. [Internet] Genebra: OMS; 2015 [acesso em 10 dez 2017]. Disponível em: http://www.who.int/violence_injury_prevention/road_safety_status/2015/ Summary_GSRRS2015_POR.pdf.

4. Andrade SSCA, de Mello-Jorge MHP. Mortalidade anos potenciais de vida perdidos por acidentes de transporte no Brasil, 2013. Rev. Saúde Pública. [Internet] 2016;50 [acesso em 5 jun 2017]. Disponível: http://dx.doi.org/10.1590/ S1518-8787.2016050006465.

5. Andrade SSCA, Jorge MHPM. Internações hospitalares por lesões decorrentes de acidente de transporte terrestre no Brasil, 2013: permanência e gastos. Epidemiol. Serv. Saúde. [Internet] 2017;26(1) [acesso em 6 jun 2017]. Disponível: http://dx.doi.org/10.5123/s1679-49742017000100004. 
6. Associação Brasileira de Prevenção de Acidentes de Trânsito. Santa Catarina terá comitê de prevenção de acidentes. [Internet] O regional sul: 2013; [acesso em 12 mai 2017]. Disponível: http://www.vias-seguras.com/a_prevencao/a_ decada_de_acoes_de_seguranca_em_cada_estado/a_decada_de_seguranca_do_transito_no_santa_catarina/santa_ catarina_tera_comite_de_prevencao_de_acidentes.

7. Ascari RA, Chapieski CM, da Silva OM, Frigo J. Perfil epidemiológico de vítimas de acidente de trânsito. Rev.Enferm. UFSM. [Internet] 2013;3(1) [acesso em 5 jun 2017]. Disponível: http://dx.doi.org/10.5902/217976927711.

8. Soares LS, de Sousa DACM, Machado ALG, da Silva GRF.Caracterização das vítimas de traumas por acidente com motocicleta internadas em um hospital público. Rev.Enferm. UERJ. [Internet] 2015;23(1) [acesso em 6 jun 2017]. Disponível: http://dx.doi.org/10.12957/reuerj.2015.15599.

9. da Silva JK, Rios MA, Rios MA, Amaral TFS, da Silva PL. Perfil dos acidentes de transporte terrestre atendidos pelo serviço de atendimento móvel de urgência. Revenferm UFPE online. [Internet] 2016;10(1) [acesso em 6 jun 2017]. Disponível: https://periodicos.ufpe.br/revistas/revistaenfermagem/article/viewFile/10915/12192.

10. Andrade SSCA, Jorge MHPM. Estimativa de sequelas físicas em vítimas de acidentes de transporte terrestre internadas em hospitais do Sistema Único de Saúde. Rev. bras. epidemiol. [Internet] 2016;19(1) [acesso em 6 jun 2017]. Disponível: http://dx.doi.org/10.1590/1980-5497201600010009.

11. Mohtasham-Amiri Z, Dastgiri S, Davoudi-kiakalyeh A, Imani A, Mollarahimi K. An epidemiological study of road traffic accidents in Guilan Province, Northern Iran in 2012. Bull.Emerg. Trauma. [Internet] 2016;4(4) [acesso em 6 jun 2017]. Disponível: https:/www.ncbi.nlm.nih.gov/pmc/articles/PMC5118576/.

12. Fernando DM, Tennakoon SU, Samaranayake AN, Wickramasinghe M. Characteristics of road traffic accident casualties admitted to a tertiary care hospital in Sri Lanka. ForensicSci. Med.Pathol. [Internet] 2017;13(1) [acesso em 6 jun 2017]. Disponível: https://dx.doi.org/ 10.1007/s12024-016-9828-3.

13. Papa MAF, Wisniewski D, Inoue KC, Molena-Fernandes CA, Évrânsito. Rev Enferm UFSM 2013 observa-se que estas aumentaram nas s entre pacientes e nora YDM, Matsuda LM. Mortalidade por acidentes de trânsito terrestre: análise comparativa.CogitareEnferm. [Internet] 2014;19(1) [acesso em 6 jun 2017]. Disponível: http://dx.doi.org/10.5380/ ce.v19i1.35934.

14. Ibrahim NA, Ajani AWO, Mustafa IA, Balogun RA, Oludara MA, Idowu OE, et al. Road traffic injury in Lagos, Nigeria: assessing prehospital care. Prehosp.Disaster Med. [Internet] 2017;32(4) [acesso em 6 jun 2017]. Disponível: https://doi.org/10.1017/S1049023X17006410.

15. Bachani AM, Hung YW, Mogere S, Akunga D, Nyamari J, Hyder AA. Helmet wearing in Kenya: prevalence, knowledge, attitude, practice and implications. PublicHealth. [Internet] 2017;144[acesso em 6 jun 2017]. Disponível: http://dx.doi.org/10.1016/j.puhe.2016.12.005.

16. Mascarenhas MDM, Souto RMCV, Malta DC, da Silva MMA, de Lima CM, Montenegro MMS. Características de motociclistas envolvidos em acidentes de transporte atendidos em serviços públicos de urgência e emergência. Ciênc. saúde coletiva. [Internet] 2016;21(12) [acesso em 6 jun 2017]. Disponível: http://dx.doi.org/10.1590/1413812320152112.24332016 .

17. de Albuquerque AM, Silva HCL, Torquato IMB, Gouveia BLA, Abrantes MSAP, Torres VSF. Vítimas de acidentes de moto com traumatismo. Rev.enferm. UFPE online. [Internet] 2016;10(5) [acesso em 6 jun 2017]. Disponível: http://bases.bireme.br/cgi-bin/wxislind.exe/iah/online/?lsisScript=iah/iah.xis\&src=google\&base=BDENF\&lang=p\&nextAction=Ink\&exprSearch=29657 \&indexSearch=ID.

18. Mascarenhas MDM, Barros MBA. Caracterização das internações hospitalares por causas externas no sistema público de saúde, Brasil, 2011. Rev. bras. epidemiol. [Internet] 2015;18(4) [acesso em 6 jun 2017]. Disponível: http://dx.doi. org/10.1590/1980-5497201500040008.

19. de Almeida RLF, Bezerra Filho JG, Braga JU, Magalhães FB, Macedo MCM, Silva KA. Via, homem e veículo: fatores de risco associados à gravidade dos acidentes de trânsito. Rev. Saúde Pública. [Internet] 2013;47(4) [acesso em 6 jun 2017]. Disponível: http://dx.doi.org/10.1590/S0034-8910.2013047003657. 
20. Gorios C, Armond JE, Rodrigues CL, Pernambuco H, Iporre RO, Colombo-Souza P. Analysis of hospitalization occurred dueto motorcycles accidents in São Paulo city. Acta ortop.bras. [Internet] 2015;23(4) [acesso em 6 jun 2017]. Disponível: http://dx.doi.org/10.1590/1413-78522015230400999.

21. Clèries $M$, Bosch $A$, Vela $E$, Bustins M. Lesiones por accidente de tráfico: aproximación desde el conjunto mínimo básico de datos de urgencias y hospitalización de agudos de Cataluña. Gac.Sanit. [Internet] 2015;29(Suppl 1) [acesso em 6 jun 2017]. Disponível: https://doi.org/10.1016/j.gaceta.2015.03.011.

22. Silva RA. Characterization of the traffic accidents and of the victims assisted by SAMU. Rev.enferm. UFPE online. [Internet] 2011;5(10) [acesso em 6 jun 2017]. Disponível: https://periodicos.ufpe.br/revistas/revistaenfermagem/article/ view/6965.

23. Seerig LM, Bacchieri G, Nascimento GG, Barros AJD, Demarco FF. Use of motorcycle in Brazil: users profile, prevalence of use and traffic accidents occurrence - a population-based study. Ciênc. saúde coletiva. [Internet] 2016;21(12) [acesso em 6 jun 2017]. Disponível: http://dx.doi.org/10.1590/1413-812320152112.28212015.

24.Cavalcante ACB, Holanda VM, Rocha CFM, Cavalcante SW, Sousa JPR, Sousa FHR. Perfil dos acidentes de trânsito atendidos por serviço pré-hospitalar móvel. Rev. Baiana de Enfermagem. [Internet] 2015;29(2) [acesso em 6 jun 2017]. Disponível: http://dx.doi.org/10.18471/rbe.v29i2.12656.

25.Rezende Neta DS, Alves AKS, Leão GM, de Araújo AA. Perfil das ocorrências de politrauma em condutores motociclísticos atendidos pelo SAMU de Teresina-PI. Rev. bras. enferm. [Internet] 2012;65(6) [acesso em 6 jun 2017]. Disponível: http://dx.doi.org/10.1590/S0034-71672012000600008.

26. Huang L, Adhikary KP, Choulagai BP, Wang N, Poudyal AK, Onta SR. Road traffic accident and its characteristics in Kathmandu Valley. J. Nepal Med. Assoc. [Internet] 2016;55(203) [acesso em 6 jun 2017]. Disponível: http://jnma.com. np/jnma/index.php/jnma/article/view/2829/2364. 